\section{What Evidence Supports Guidelines for Use of ACE Inhibitors and ARBs in Diabetes?}

In this issue of JMCP, Cooke and Fatodu inform us that of 1,698 patients with diabetes, $13 \%(n=215)$ had a medical claim indicating renal involvement. ${ }^{1}$ In the subset of 215 diabetes patients with renal involvement, 177 had at least 1 medical claim with a diagnosis of hypertension, and the use of angiotensin-converting enzyme (ACEs) inhibitors or angiotensin receptor blockers (ARBs) was high $(85.3 \%, \mathrm{n}=151)$. In the subgroup of 38 diabetes patients with renal involvement but without hypertension, the use of ACE inhibitors or ARBs was significantly lower ( $47 \%, \mathrm{n}=18, P<0.001$ ). Overall, these administrative claims data for the dates of service from April 1 , 2001, through March 31, 2002, revealed that 915 (53.9\%) of the diabetes patients had at least 1 claim for either an ACE inhibitor or ARB. Relying on the 2002 and 2004 position statements of the American Diabetes Association on hypertension management in adults with diabetes, ${ }^{2}$ Cooke and Fatodu suggest that diabetes patients are undertreated with ACE inhibitors or ARBs. However, for their subgroup of patients with diabetes and hypertension ( $\mathrm{n}=1,072,63.1 \%)$, more than 4 out of 5 ( $\mathrm{n}=951,85.4 \%$ ) were treated with an ACE inhibitor or ARB, and the authors acknowledge that only 20 diabetes patients (1.2\% overall) who did not have hypertension but did have evidence of renal involvement did not receive either an ACE inhibitor or ARB.

While Cooke and Fatodu have support from clinical practice guidelines to claim undertreatment of diabetes patients with ACE inhibitors or ARBs, some scientists have recently questioned the evidence to support these guidelines. The results of a systematic review and meta-analysis performed last year for studies published through January 2005 refute the assumption that ACE inhibitors and ARBs, collectively renin-angiotensin system (RAS) inhibitors, have renoprotective effects that extend beyond reduction in blood pressure. ${ }^{3}$ Casas et al. concluded that not only are the additional renoprotective actions of ACE inhibitors and ARBs beyond lowering blood pressure unproven in persons with diabetes, there is not sufficient evidence to conclude that there is renoprotection from these drugs in nondiabetic patients with renal disease.

Specifically, Casas et al. found a relative risk of 0.71 for doubling of serum creatinine for the RAS inhibitors, but the 95\% confidence interval $(\mathrm{CI}, 0.49-1.04)$ crossed 1.0 (i.e., no risk reduction), and a small benefit on end-stage renal disease (ESRD, relative risk, 0.87; 95\% CI, 0.75-0.99). When Casas et al. analyzed the results by study population size, there was a smaller benefit in large studies. For patients with diabetic nephropathy, there was no benefit for RAS inhibitors by the measure of a 2-fold increase in serum creatinine (relative risk, $1.09 ; 95 \% \mathrm{CI}, 0.55-2.15)$ and no benefit in progression to ESRD (relative risk, 0.89; 95\% CI, 0.74-1.07), glomerular filtration rate, or absolute creatinine amounts. This systematic review and meta-analysis of data from 13 studies generated a firestorm response, generally focused on the heavy reliance on the results of the Antihypertensive and Lipid-Lowering Treatment to Prevent Heart Attack Trial (ALLHAT). ${ }^{4}$ Casas and coauthors pointed out in rebuttal to the letters that the ALLHAT results did contribute about half of the available evidence on renal outcomes, but ALLHAT included renal disease as a prespecified outcome, and ALLHAT is the only study with a population size near the size necessary $(57,000)$ to demonstrate a $10 \%$ relative risk reduction in ESRD. ${ }^{5}$

Aside from the specific question regarding the potential value of ACE inhibitors or ARBs in renoprotection beyond blood pressure reduction, the results of key clinical trials suggested that there might be some effect of ACE inhibitors on glucose metabolism and a potential role in diabetes prevention. Two studies in particular, neither of which was designed to assess specifically the outcome of a new diagnosis of diabetes, suggested that ACE inhibitors might be associated with a side effect in preventing diabetes. Ingelfinger and Solomon in an editorial published earlier this month ${ }^{6}$ inform us that the Captopril Prevention Project (CAPPP) found a 14\% lower incidence of diabetes in the captopril group compared with diuretics or betablockers in hypertensive patients, ${ }^{7}$ and results of the Heart Outcomes Prevention Evaluation (HOPE) Study in patients at high risk for cardiovascular events found a 34\% reduction in risk of newly diagnosed diabetes in patients who received ramipril $10 \mathrm{mg}$ per day compared with placebo. ${ }^{8}$ However, the absolute rates of a new diagnosis of diabetes were small. For the 5 years of follow-up in the HOPE Study, 102 (3.6\%) patients in the ramipril arm developed a new diagnosis for diabetes versus 155 (5.4\%) for placebo. In a study designed specifically to assess the development of a new diagnosis of diabetes in patients with either impaired glucose tolerance or impaired fasting glucose levels, the Diabetes Reduction Assessment with Ramipril and Rosiglitazone Medication (DREAM) results showed no difference in new diagnosis of diabetes for an average 3 years of therapy with ramipril (up to $15 \mathrm{mg}$ per day), $17.1 \%$ versus $18.5 \%$ for placebo, (hazard ratio [HR], 0.91; 95\% CI, 0.80-1.03). ${ }^{9}$

The choice of a preferred antihypertensive agent in a particular patient involves consideration of multiple factors. ${ }^{10}$ ALLHAT results showed that compared with the diuretic chlorthalidone, the ACE inhibitor lisinopril was associated with a higher risk of stroke $(P=0.02)$ and a higher risk of cardiovascular disease $(P$ $<0.001$ ), including a higher risk of heart failure and higher risk of coronary revascularization. ${ }^{11}$ The Valsartan Antihypertensive Long-term Use Evaluation (VALUE) trial involving 15,245 patients at high risk for cardiac events, including $31.7 \%$ with diabetes, found no difference in the primary composite outcome of sudden cardiac death, fatal myocardial infarction (MI), cardiovascular death, or cardiovascular morbidity (including heart failure) between the ARB valsartan and amlodipine. However, valsartan had a smaller effect compared with amlo- 
dipine on blood-pressure lowering and had an increased risk of MI (HR, 1.19; $P<0.02) .^{12}$

Two major trials have compared ACE inhibitors to ARBs in cardiovascular outcomes. The Evaluation of Losartan In The Elderly (ELITE) II trial showed that the ARB losartan was not superior to the ACE inhibitor captopril in reducing morbidity and mortality in patients with heart failure, and the HR for sudden death was close to significant (HR, 1,30; 95\% CI, 1.001.69) for losartan versus captopril. ${ }^{13}$ The Optimal Trial in Myocardial Infarction with the Angiotensin II Antagonist Losartan (OPTIMAAL) also failed to show an advantage for ARBs over ACE inhibitors; in fact, most end points showed a trend in favor of captopril over losartan, with cardiovascular death significantly lower in the captopril group. ${ }^{14}$

Until more evidence accumulates on the alleged renoprotection associated with RAS inhibition, it seems reasonable for clinicians to not use pharmacologic intervention with ACE inhibitors or ARBs in normotensive patients with diabetes. For hypertensive patients with diabetes, prescribing a thiazide diuretic would also seem to represent the practice of evidence-based medicine. On the other hand, a combination product containing hydrochlorothiazide (HCTZ) and an ACE inhibitor such as benazepril or lisinopril has a managed care price before member cost share of about $\$ 0.70$ per day or $\$ 21$ per month compared with about $\$ 0.50$ per day or $\$ 15$ per month with either benazepril or lisinopril alone. ${ }^{15}$ While we await evidence of a renoprotective effect of RAS inhibition, the combination product of HCTZ and an ACE inhibitor appears to be a good investment to produce desired clinical outcomes in hypertensive patients with diabetes.

\section{Frederic R. Curtiss, $\mathrm{PhD}, \mathrm{RPh}, \mathrm{CEBS}$ Editor-in-Chief fcurtiss@amcp.org}

morbidity and mortality in hypertension: The Captopril Prevention Project (CAPPP) randomised trial. Lancet. 1999;353:611-16.

8. The Heart Outcomes Prevention Evaluation Study investigators. Effects of an angiotensin-converting-enzyme inhibitor, ramipril, on cardiovascular events in high-risk patients. N Engl J Med. 2000;342:145-53.

9. The DREAM Trial investigators. Effect of ramipril on the incidence of diabetes. N Engl J Med. 2006;355:1551-62.

10. Bethel MA, Feinglos MN. Pharmacological management of the patient with newly diagnosed type 2 diabetes. J Clin Outcomes Manag. 2006;13(7): 392, 398-403.

11. The ALLHAT Officers and Coordinators for the ALLHAT Collaborative Research Group. The Antihypertensive and Lipid-Lowering Treatment to Prevent Heart Attack Trial: major outcomes in high-risk hypertensive patients randomized to angiotensin-converting enzyme inhibitor or calcium channel blocker vs diuretic: the Antihypertensive and Lipid-Lowering Treatment to Prevent Heart Attack Trial (ALLHAT). JAMA. 2002;288:2981-97.

12. Julius S, Kjeldsen SE, Weber Mfor the VALUE trial group. Outcomes in hypertensive patients at high cardiovascular risk treated with regimens based on valsartan or amlodipine: the VALUE randomised trial. Lancet 2004; 363:2022-31.

13. Pitt B, Poole-Wilson PA, Segal R, et al. Effect of losartan compared with captopril on mortality in patients with symptomatic heart failure: randomised trial-the Losartan Heart Failure Survival Study ELITE II. Lancet. 2000; 355:1582-87.

14. Dickstein K, Kjekshus J, and the OPTIMAAL Steering Committee. Effects of losartan and captopril on mortality and morbidity in high-risk patients after acute myocardial infarction: the OPTIMAAL randomised trial. Lancet. 2002; 360:752-60.

15. Data search performed October 6, 2006, of the data warehouse of a national pharmacy benefits manager representing approximately 500,000 beneficiaries of small employer drug benefit plans for pharmacy claims with dates of service from July 1, 2006, through September 30, 2006.

\section{REFERENCES}

1. Cooke CE, Fatodu H. Physician conformity and patient adherence to ACE inhibitors and ARBs in patients with diabetes, with and without hypertension, in a Medicaid managed care organization. J Manag Care Pharm. 2006;8:649-55.

2. American Diabetes Association. Hypertension management in adults with diabetes. Diabetes Care. 2004;(27)(suppl 1):S65-S67.

3. Casas JP, Chua W, Loukogeorgakis S, et al. Effect of inhibitors of the reninangiotensin system and other antihypertensive drugs on renal outcomes: systematic review and meta-analysis. Lancet. 2005;366:2026-33.

4. Adler A, de Zeeuw D, Lewis EJ, et al. Renoprotective effects of reninangiotensin-system inhibitors (letter). Lancet. 2006;366:897-900.

5. Casas JP, Vallance P, Smeeth L, Hingorani AD, MacAllister RJ. Renoprotective effects of renin-angiotensin-system inhibitors (letter-authors reply). Lancet. 2006;366:900-02.

6. Ingelfinger JR, Solomon CG. Angiotensin-converting-enzyme inhibitors for impaired glucose toleration-is there still hope? N Engl J Med. 2006;355: 1608-10.

7. Hansson L, Lindholm LH, Niskanen L, et al. Effect of angiotensin-converting-enzyme inhibition compared with conventional therapy on cardiovascular 